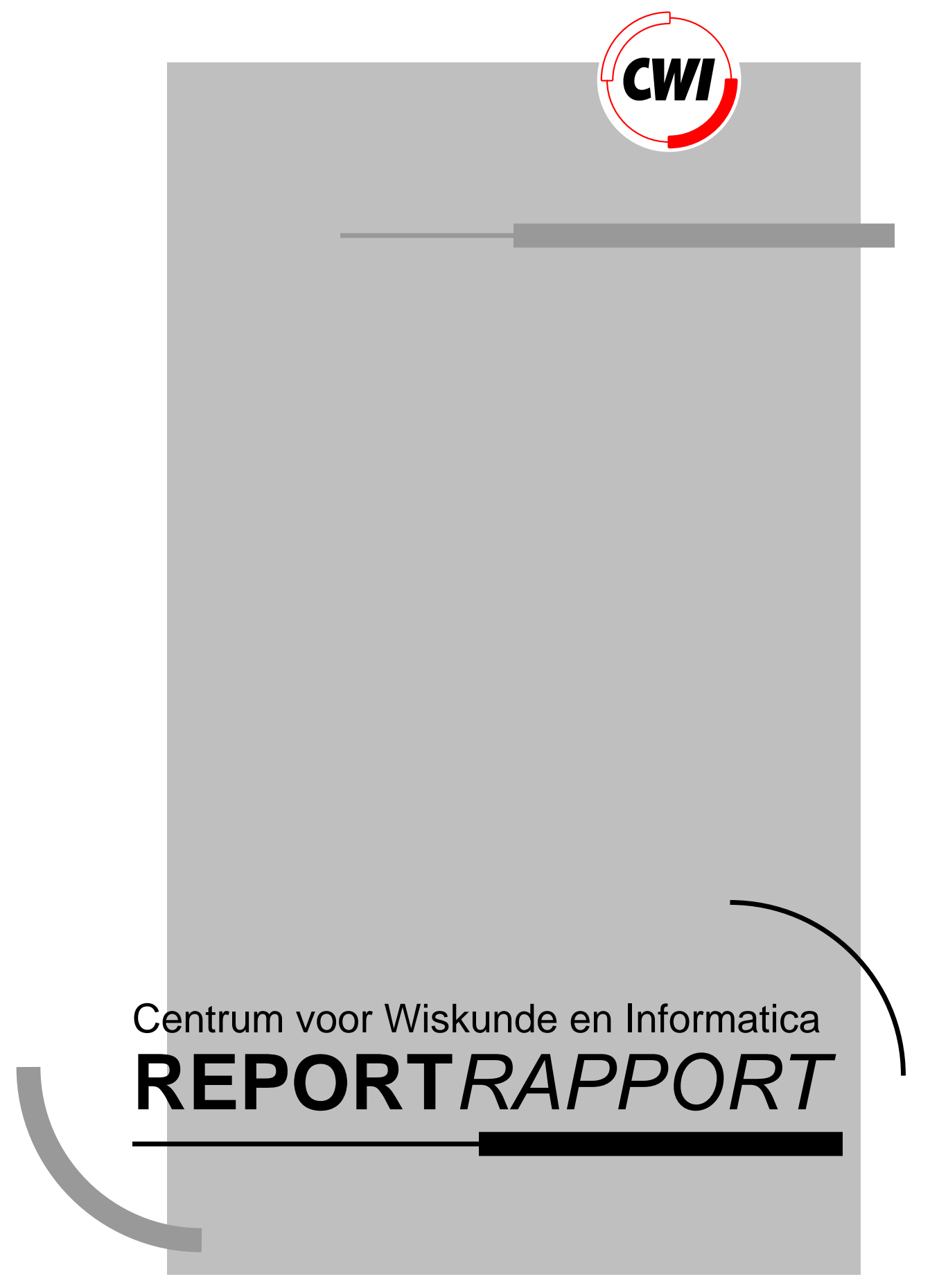

On the construction of morphological operators which re selfdual and activity-extensive

H.J.A.M. Heijmans

Department of Operations Reasearch, Statistics, and System Theory

BS-R9307 1993 



\title{
On the Construction of Morphological Operators \\ which are Selfdual and Activity-extensive
}

\author{
Henk J.A.M. Heijmans \\ $C W I$ \\ P.O. Box 4079, 1009 AB Amsterdam, The Netherlands
}

\begin{abstract}
This paper presents a systematic method to construct selfdual increasing morphological operators on $\mathcal{P}\left(\mathbb{Z}^{d}\right)$ using the concept of a switch operator. Furthermore, it shows how to modify a selfdual operator in such a way that the sequence of iterates of the resulting modified operator converges pointwise monotone to a selfdual morphological filter. The approach is based on the concept of the centre operator and the activity ordering.
\end{abstract}

AMS 1991 Mathematics Subject Classification: 68U10

Keywords \& Phrases: mathematical morphology, morphological filter, iteration, centre, finite window operator, activity ordering, activity-extensive operator, selfdual operator, pointwise monotone, switch operator.

Note: Paper presented at the Workshop "Mathematical Morphology and its Applications to Signal Processing", 12-14 May 1993, Barcelona, Spain

\section{Introduction}

An important problem in mathematical morphology is the design of morphological filters, i.e. operators which are increasing and idempotent. The idempotence property is desirable, e.g. when the operator is used for noise cleaning; it guarantees that a second or third application of the operator is superfluous in the sense that it does not have any further effect on the outcome. A general theory of morphological filters has been developed by Matheron and Serra [7]; some of their results are discussed below. Recently, Heijmans and Serra [3] have shown how to construct morphological filters by iteration; see also [1, 4, 6].

The median operator (see Sect. 4 for a definition) is the prototype of a morphological operator which is selfdual. Selfduality of an operator means that it treats fore- and background in an analogous way; with respect to noise cleaning applications, selfduality is a useful property. Unfortunately, the median operator is not idempotent; even worse, iteration of the median operator may lead to oscillations. The purpose of this paper is to characterize the class of selfdual increasing operators (to that goal we shall introduce the concept of a 'switch operator') and to explain how to modify such operators in order that their iterates converge to a morphological filter. We use a result by Serra [7] which says that the iterates of the centre of an overfilter and an underfilter form an increasing sequence in the sense of the activity ordering.

For the reader's convenience we restrict ourselves to binary images throughout this paper; for a general account in the framework of complete lattices the reader may refer to our book [2].

In Sect. 2 we introduce some terminology and in Sect. 3 we explain briefly how to obtain filters by iteration. In Sect. 4 we present some basic selfdual operators. A general construction procedure for such operators is outlined in Sect. 5. Finally, in Sect. 6 we explain how to modify such operators so that they become convergent under iteration. 


\section{Preliminary notions}

Throughout this paper we restrict attention to increasing operators on $\mathcal{P}\left(\mathbb{Z}^{d}\right)$. Openings will be denoted by $\alpha$ and closings by $\beta$. The negative of the operator $\psi$, denoted by $\psi^{*}$, is

$$
\psi^{*}(X)=\left[\psi\left(X^{\mathrm{c}}\right)\right]^{\mathrm{c}}, X \subseteq \mathbb{Z}^{d}
$$

where $X^{\mathrm{c}}$ is the complement of $X$.

2.1. Definition. An increasing operator is called

(a) an overfilter if $\psi^{2} \geq \psi$;

(b) an underfilter if $\psi^{2} \leq \psi$;

(c) an inf-overfilter if $\psi($ id $\wedge \psi)=\psi$;

(d) a sup-underfilter if $\psi($ id $\vee \psi)=\psi$;

(e) a strong filter if $\psi$ is both a sup-filter and an inf-filter.

The results stated below can be found in [7].

2.2. Definition. Let $\phi, \psi$ be increasing operators. We say that $\phi$ is less active than $\psi$, denoted by $\phi \preceq \psi$, if

$$
\text { id } \wedge \phi \geq \text { id } \wedge \psi \text { and id } \vee \phi \leq \text { id } \vee \psi
$$

Serra [7] has proved that the family of operators is a complete lattice under the activity ordering; see also [5]. The activity infimum of a set of operators $\left\{\psi_{i} \mid i \in i\right\}$, denoted by $\curlywedge_{i \in I} \psi_{i}$, is called the centre of $\psi_{i}$ and is given by

$$
\gamma=\left(\mathrm{id} \wedge \bigvee_{i \in I} \psi_{i}\right) \vee \bigwedge_{i \in I} \psi_{i}
$$

If every $\psi_{i}$ is increasing, then $\gamma$ is such as well. If $\phi, \psi$ are increasing and $\phi \leq \psi$ then their centre is

$$
\gamma=(\text { id } \wedge \psi) \vee \phi=(\text { id } \vee \phi) \wedge \psi
$$

Note that $\gamma \preceq \phi, \psi$.

2.3. Definition. The operator $\psi$ is called activity-extensive if $\psi^{n} \preceq \psi^{n+1}$ for every $n \geq 0$.

It is evident that every extensive or anti-extensive operator is activity-extensive. The operator $\psi$ is activity-extensive if, for every set $X$, the sequence $\psi^{n}(X)$ is pointwise monotone. This means that for every point $x \in \mathbb{Z}^{d}$ the value $\psi^{n}(X)(x)$ (which is 1 if $x$ lies in $\psi^{n}(X)$ and 0 otherwise) changes at most once. For example, $0,0, \cdots, 0,1,1, \cdots$ is an admitted sequence as well as $1,1, \cdots, 1,0,0, \cdots$. However $0,0, \cdots, 0,1,1,0,0, \cdots$ is a prohibited sequence.

\section{Iteration}

It is possible to construct morphological filters by iteration of arbitrary increasing morphological operators $[1,3,6]$. Here we discuss only a special case. We say that a sequence $X_{n} \subseteq \mathbb{Z}^{2}$ converges to $X$, written $X_{n} \rightarrow X$, if

(i) $h \in X_{n_{k}}$ for some subsequence $n_{k}$ implies $h \in X$;

(ii) if $h \in X$ then $h \in X_{n}$ for $n$ sufficiently large.

An increasing morphological operator $\psi$ is called a finite window operator if

$$
h \in \psi(X) \Longleftrightarrow h \in \psi(X \cap W(h)),
$$

for $h \in \mathbb{Z}^{d}$ and $X \subseteq \mathbb{Z}^{d}$. Here $W(h)$ is a finite subset of $\mathbb{Z}^{d}$ for every $h \in \mathbb{Z}^{d}$. If $\psi$ is translation invariant then $W(h)=W_{h}$ for some finite set $W$. An operator using finitely many finite structuring elements is a finite window operator.

3.1. Theorem. Let $\psi, \psi^{\infty}$ be increasing finite window operators and assume that $\psi^{n} \rightarrow \psi^{\infty}$ (this means that $\psi^{n}(X) \rightarrow \psi^{\infty}(X)$ for every $\left.X \subseteq \mathbb{Z}^{d}\right)$, then $\psi^{\infty}$ is a morphological filter. 
Refer for a proof of the following result to [7]; see also [2]. If $\phi$ is an inf-overfilter and $\psi$ a sup-underfilter with $\phi \leq \psi$, then there exists a strong filter $\omega$ with $\phi \leq \omega \leq \psi$; one calls $\omega$ the middle filter. Maisonneuve [4] showed that transfinite iteration of the centre $\gamma$ of $\phi$ and $\psi$ yields $\omega$. But in our case the situation is much simpler.

3.2. Proposition. Assume $\phi \leq \psi$ are finite window operators; assume also that $\phi$ is an inf-overfilter and that $\psi$ is a sup-underfilter. The centre $\gamma=(\mathrm{id} \wedge \psi) \vee \phi$ is activity-extensive and $\gamma^{n}$ converges to the strong middle filter $\omega$.

Assume that $\alpha \leq \psi$ is an opening; it is easy to check that $\psi \alpha$ is an inf-overfilter. Dually, $\psi \beta$ is a sup-underfilter if $\beta \geq \psi$ is a closing. We arrive at the following result.

3.3. Proposition. Let $\psi$ be an increasing operator; if $\alpha \leq \psi$ is an opening and $\beta \geq \psi$ is a closing, then

$$
\pi=(\text { id } \wedge \psi \beta) \vee \psi \alpha
$$

is activity-extensive and $\pi \preceq \psi$. Furthermore, if $\alpha^{\prime} \leq \alpha$ and $\beta^{\prime} \geq \beta$ are an opening and a closing respectively and if $\pi^{\prime}=\left(\right.$ id $\left.\wedge \psi \beta^{\prime}\right) \vee \psi \alpha^{\prime}$ then $\pi^{\prime} \preceq \pi$.

\section{Selfdual operators}

The interest in selfdual operators stems from the fact that such operators treat foreground and background of an image identically. Let $A \subseteq \mathbb{Z}^{d}$ be a finite structuring element with $n$ points $a_{1}, a_{2}, \cdots, a_{n}$ and let $X \subseteq \mathbb{Z}^{d}$ be a binary image. The rank operator $\rho_{A, s}(1 \leq s \leq n)$ in a point $h$ is computed as follows: put the values $X\left(a_{i}+h\right)$ in decreasing order and pick the value at the $s^{\prime}$ th position. It is easy to see that $\rho_{A, 1}(X)=X \oplus \check{A}$, that $\rho_{A, n}(X)=X \ominus A$ and that

$$
\rho_{A, n} \leq \rho_{A, n-1} \leq \cdots \leq \rho_{A, 1}
$$

Furthermore $\rho_{A, s}^{*}=\rho_{A, n+1-s}$. If $n$ is odd and $s=(n+1) / 2$ then $\rho_{A, s}$ is selfdual; it is called the median operator and denoted by $\mu_{A}$.

A general way to obtain selfdual operators is provided by the next result.

4.1. Proposition. Let $\psi$ be an increasing operator. The centre of $\psi \wedge \psi^{*}$ and $\psi \vee \psi^{*}$ given by

$$
\gamma=\left(\text { id } \wedge\left(\psi \vee \psi^{*}\right)\right) \vee\left(\psi \wedge \psi^{*}\right)
$$

is selfdual. Furthermore $\gamma \preceq \psi$ and $\gamma \preceq \psi^{*}$. Moreover, if $\psi$ is a filter then $\gamma$ is activity-extensive.

Let $A \subseteq \mathbb{Z}^{d}$ be a structuring element with $n$ points. If $2 s \geq n+1$ then the rank operator $\rho_{A, s}$ has negative $\rho_{A, n-s+1}$ and $\rho_{A, s} \leq \rho_{A, n-s+1}$; in this case

$$
\psi_{s}=\left(\mathrm{id} \wedge \rho_{A, n-s+1}\right) \vee \rho_{A, s}
$$

is a selfdual operator. This operator extends the median operator: if $n$ is odd and $s=(n+1) / 2$ then $\psi_{s}=\mu_{A}$. Note that $\psi_{s} \preceq \psi_{t}$ if $s \geq t$ and $2 s, 2 t \geq n+1$; furthermore $\psi_{n}=$ id if $0 \in A$.

\section{Switch operators}

This section discusses a systematic method to construct increasing selfdual operators on $\mathcal{P}\left(\mathbb{Z}^{d}\right)$. Proofs can be found in [2]. The following definition plays a central role.

5.1. Definition. An anti-extensive operator $\sigma: \mathcal{P}\left(\mathbb{Z}^{d}\right) \rightarrow \mathcal{P}\left(\mathbb{Z}^{d}\right)$ is called a switch operator if it satisfies (S1) $\sigma(Y) \cap X \subseteq \sigma(X)$ if $X \subseteq Y$;

(S2) $\sigma(X \cup\{h\}) \cap \sigma\left(X^{\mathrm{c}} \cup\{h\}\right)=\varnothing$, for $h \in \mathbb{Z}^{d}, X \subseteq \mathbb{Z}^{d}$. 
Before we explain these conditions we present an example. Let $\mu$ be the median operator with the rhombus (origin and its 4-neighbours) as structuring element. If a point $h \in X$ is surrounded by at least three 4-neighbours which are not contained in $X$, like in one of the following figures

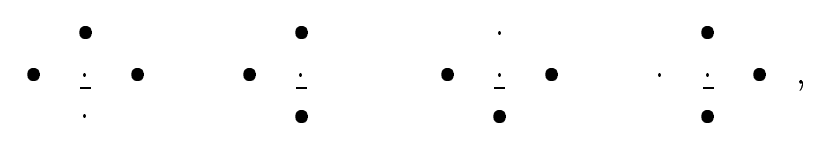

then $h \notin \mu(X)$. Define $\sigma(X)$ as the points $h \in X$ which are not contained in $\mu(X)$; this means that one of these four configurations centred at $h$ lies entirely in $X^{\mathrm{c}}$. Dually, if $h$ is a background pixel, i.e., $h \in X^{\mathrm{c}}$, then $h \in \mu(X)$ if at least three of its 4-neighbours belong to $X$. In that case $h \in \sigma\left(X^{\mathrm{c}}\right)$. Thus application of $\sigma$ to $X$ yields all points in $X$ which are deleted by $\mu$ and application of $\sigma$ to $X^{\mathrm{c}}$ yields all points in $X^{\mathrm{c}}$ which are added by $\mu$. In other words, $\sigma(X)$ and $\sigma\left(X^{\mathrm{c}}\right)$ represent the pixels whose state switches from 1 to 0 and from 0 to 1 respectively.

Condition (S1) expresses that a point $h$ in $Y$ which switches by application of $\sigma$ to $Y$, also switches if $\sigma$ is applied to a smaller set $X$ which contains $h$. Condition (S2) guarantees that two complementary configurations centred at a point $h$ cannot both force a switch of $h$; note that the fact that $\sigma$ is antiextensive gives that the intersection in (S2) contains at most the point $h$.

5.2. Proposition. With every increasing, selfdual operator $\psi$ there corresponds a unique switch operator $\sigma$ and vice versa. The operators $\psi$ and $\sigma$ are interrelated by the formulas:

$$
\begin{gathered}
\psi(X)=(X \backslash \sigma(X)) \cup \sigma\left(X^{\mathrm{c}}\right) ; \\
\sigma(X)=X \cap \psi\left(X^{\mathrm{c}}\right) .
\end{gathered}
$$

The next result provides a complete characterization of translation invariant switch operators.

5.3. Proposition. Let $\mathcal{A} \subseteq \mathcal{P}\left(\mathbb{Z}^{d}\right)$ be a collection of structuring elements with the property that

$$
0 \notin A \text { and } A \cap B \neq \varnothing \text { for } A, B \in \mathcal{A}
$$

then

$$
\sigma_{\mathcal{A}}(X)=X \cap\left[\bigcup_{A \in \mathcal{A}} X^{\mathrm{c}} \ominus A\right]
$$

is a switch operator. Conversely, if $\sigma$ is a switch operator and

$$
\mathcal{A}=\left\{X^{\mathrm{c}} \mid 0 \in \sigma(X)\right\}
$$

then $\mathcal{A}$ obeys condition (5.3) and $\sigma=\sigma_{\mathcal{A}}$.

If $\psi$ is an increasing, selfdual operator and $\sigma$ the associated switch operator given by (5.2), then the set $\mathcal{A}$ in $(5.5)$ is

$$
\mathcal{A}=\{A \in \mathcal{V}(\psi) \mid 0 \notin A\}
$$

Note that $A \cap B \neq \varnothing$ if $A, B \in \mathcal{V}(\psi)$. For assume that $A \cap B=\varnothing$, then $A \subseteq B^{\mathrm{c}}$, so $0 \in \psi(A) \subseteq \psi\left(B^{\mathrm{c}}\right)=$ $(\psi(B))^{\mathrm{c}}$. However, $0 \in \psi(B)$, a contradiction.

Most of the structuring elements in (5.6) are redundant. For example, if $A, B \in \mathcal{V}(\psi)$ and $A \subseteq B$, then $B$ can be omitted.

5.4. Examples. (a) Let $\mu$ be the median operator on $\mathcal{P}\left(\mathbb{Z}^{2}\right)$ with the rhombus as structuring element. It is easy to verify that $\psi$ is of the form (5.1), where $\sigma=\sigma_{\mathcal{A}}$ and $\mathcal{A}$ contains the structuring elements:

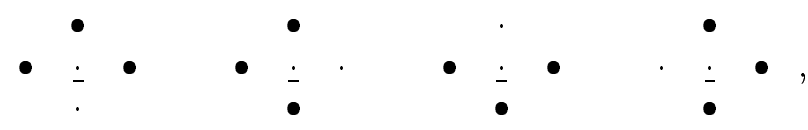

In fact, it is obvious that every element in the kernel of $\mu$ which does not contain the origin must include one of these four configurations. 
(b) Suppose that $\mathcal{A}$ contains only one element, namely

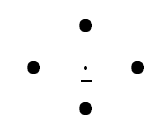

Thus a point in $X$ is deleted by $\sigma_{\mathcal{A}}$ if all 4-neighbours lie in the complement of $X$. In this case the associated selfdual operator is

$$
\psi_{4}=\left(\text { id } \wedge \rho_{A, 2}\right) \vee \rho_{A, 4}
$$

defined in Sect. 4; here $A$ is the rhombus structuring element.

5.5. Proposition. Given a switch operator $\sigma$ and an increasing extensive operator $\eta$, then $\sigma^{\prime}=\sigma \eta \wedge$ id is a switch operator as well and $\sigma^{\prime} \leq \sigma$. Let $\psi, \psi^{\prime}$ be the selfdual operators associated with $\sigma, \sigma^{\prime}$ respectively, by means of relation (5.1). Then

$$
\psi^{\prime}=(\text { id } \wedge \psi \eta) \vee \psi \eta^{*}
$$

in other words, $\psi^{\prime}$ is the centre of $\psi \eta^{*}$ and $\psi \eta$.

Let $\sigma, \sigma^{\prime}$ be switch operators on $\mathcal{P}\left(\mathbb{Z}^{d}\right)$ and let $\psi, \psi^{\prime}$ be the corresponding selfdual operators given by (5.1), then

$$
\sigma \leq \sigma^{\prime} \Longleftrightarrow \psi \preceq \psi^{\prime}
$$

\section{Towards activity-extensivity}

Suppose that $\psi$ is a selfdual operator on $\mathcal{P}\left(\mathbb{Z}^{d}\right)$. If $\alpha \leq \psi$ is an opening and if $\beta=\alpha^{*}$ is the negative closing, then $\beta \geq \psi^{*}$. The operator

$$
\pi=(\text { id } \wedge \psi \beta) \vee \psi \alpha
$$

is selfdual and activity-extensive by Prop. 3.3. Furthermore,

$$
\pi \preceq \psi
$$

and the larger $\alpha$ the more active $\pi$. Proposition 3.2 gives that the sequence $\pi^{n}$ converges to the middle filter $\omega$ of $\psi \alpha$ and $\psi \beta$, presumed that $\psi, \alpha, \beta$ are finite window operators. This filter $\omega$ is strong and selfdual.

The most active $\psi$ of this form is

$$
\pi=(\text { id } \wedge \psi \hat{\psi}) \vee \psi \check{\psi}
$$

where $\check{\psi}$ is the lower envelope and $\hat{\psi}$ the upper envelope; see [7]. It is not possible in general to find closed expressions for $\check{\psi}$ and $\hat{\psi}$. To get an opening $\alpha \leq \psi$ one must look for structuring elements $A \in \operatorname{lnv}($ id $\wedge \psi)$. In fact, if $\mathcal{M} \subseteq \operatorname{lnv}($ id $\wedge \psi)$, then $\alpha$ given by

$$
\alpha(X)=\bigcup_{A \in \mathcal{M}} X \circ A
$$

satisfies $\alpha \leq \psi$. The next lemma explains how to find elements of $\operatorname{Inv}(\mathrm{id} \wedge \psi)$ when the switch operator associated with $\psi$ is known.

6.1. Lemma. Let $\psi$ be a selfdual increasing operator on $\mathcal{P}\left(\mathbb{Z}^{d}\right)$ and let $\sigma$ be the corresponding switch operator, then

$$
\operatorname{lnv}(\text { id } \wedge \psi)=\left\{A \subseteq \mathbb{Z}^{d} \mid \sigma(A)=\varnothing\right\} .
$$

The results obtained in this chapter suggest the following general construction method for selfdual (strong) filters. 
6.2. Corollary. Let $\sigma$ be a switch operator on $\mathcal{P}\left(\mathbb{Z}^{d}\right)$ and let $\psi$ be the corresponding selfdual operator. Assume that $\psi$ is continuous (this holds, e.g., if $\sigma$ uses only finite structuring elements). Let $A_{1}, A_{2}, \cdots, A_{p}$ be finite structuring elements which satisfy $\sigma\left(A_{j}\right)=\varnothing$. If $\alpha$ is the opening $\alpha(X)=\bigcup_{j=1}^{p} X \circ A_{j}$ and if $\beta$ is the negative closing, then the operator

$$
\pi=(\text { id } \wedge \psi \beta) \vee \psi \alpha
$$

is selfdual and activity-extensive; the sequence $\pi^{n}$ converges to a strong selfdual filter.

We conclude with some examples.

6.3. Examples. (a) Let $\mu$ be the median operator associated with the rhombus. The $2 \times 2$ square $A$ is invariant under $\mu$. Let $\alpha$ be the structural opening with $A$ and $\beta=\alpha^{*}$. The theory above gives that $\pi=($ id $\wedge \mu \beta) \vee \mu \alpha$ is selfdual and activity-extensive and that $\pi \preceq \mu$.

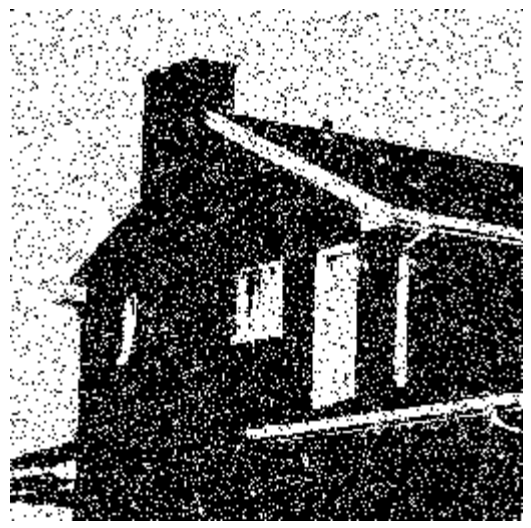

(a)

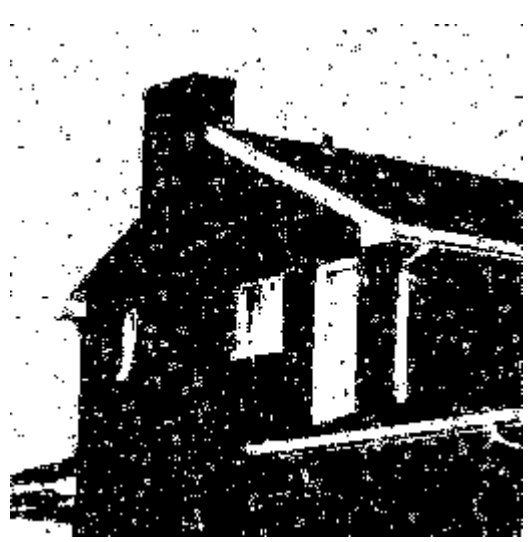

(c)

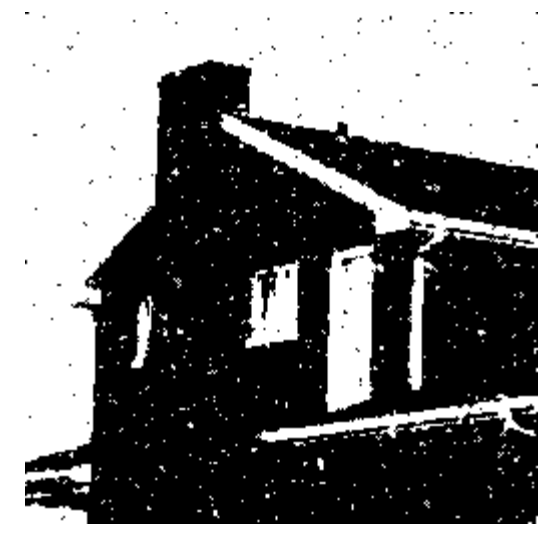

(b)

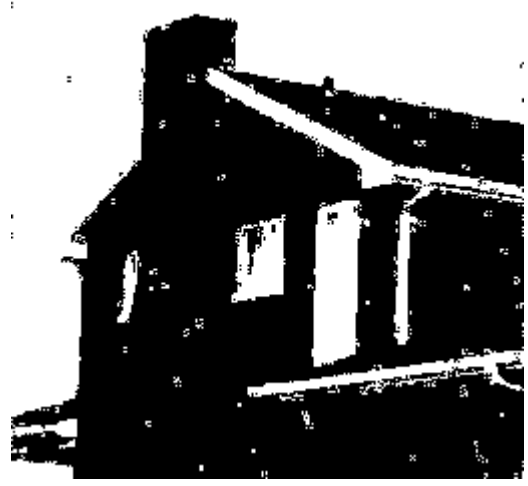

(d)

FIGURE 1. Iterates of $\pi=(\mathrm{id} \wedge \mu \beta) \vee \mu \alpha$ where $\mu$ is the median operator associated with the rhombus, $\alpha$ is the $2 \times 2$ opening and $\beta$ the dual closing. (a) Original image; (b) median; (c) 1 iteration of $\pi$; (d) $\infty$ iterations of $\pi$.

(b) Some of the ideas in this section can be traced back to an interesting unpublished paper by Maisonneuve [4]. Here we briefly discuss an example from that paper. Consider the hexagonal grid; let $\mathcal{A}$ comprise the configurations

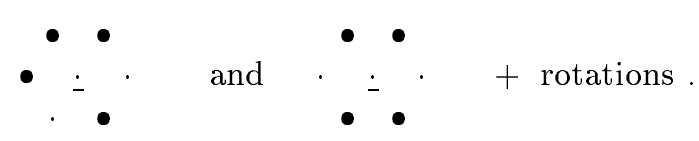


The operator $\psi$ associated with $\sigma_{\mathcal{A}}$ resembles the median operator $\mu$ associated with the elementary hexagon. However, to obtain $\mu$ one has to add

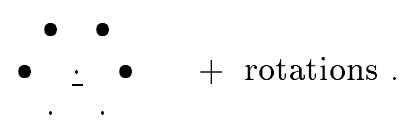

to the set $\mathcal{A}$. In particular, the operator $\psi$ is less active than $\mu$. It is easy to see that $\psi$ is not activityextensive. To obtain a modification of $\psi$ which is activity-extensive, one has to find one or more structuring elements in $\ln v($ id $\wedge \psi)$. It is evident that the triangles

$$
T_{1}=\bullet \bullet \quad, \quad T_{2}=\bullet \bullet
$$

satisfy this property. If $\alpha_{T}=\alpha_{T_{1}} \vee \alpha_{T_{2}}$ and $\beta_{T}=\alpha_{T}^{*}$, then $\pi=$ (id $\left.\wedge \psi \beta_{T}\right) \vee \psi \alpha_{T}$ is increasing, selfdual and activity-extensive; furthermore, $\pi$ is a finite window operator. Consequently, $\pi^{n}$ converges to a strong selfdual filter which we call the Maisonneuve filter after its inventor.

(c) Our final example concerns a modification of the median operator $\mu$ associated with the $3 \times 3$ square. Let $\mathcal{A}$ consist of the structuring elements

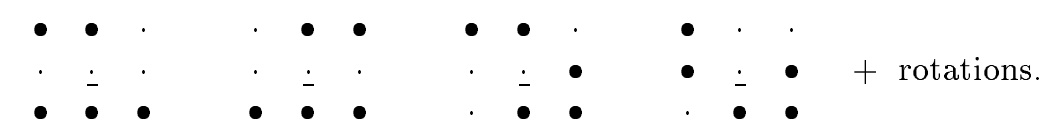

It is obvious that the selfdual operator $\psi$ associated with the switch operator $\sigma_{\mathcal{A}}$ is less active than $\mu$. To obtain a modification of $\psi$ which is selfdual one has to find a structuring element $C$ so that $\sigma_{\mathcal{A}}(C)=\varnothing$. It is easy to see that the $2 \times 2$ square is a solution.

\section{References.}

[1] H. J. A. M. Heijmans. Iteration of morphological transformations. CWI Quarterly, 2:19-36, 1989.

[2] H. J. A. M. Heijmans. Morphological Image Operators. Academic Press, in preparation.

[3] H. J. A. M. Heijmans and J. Serra. Convergence, continuity and iteration in mathematical morphology. Journal of Visual Communication and Image Representation, 3:84-102, 1992.

[4] F. Maisonneuve. Ordinaux transfinis et sur- (ou sous-) potentes. Technical report N780, Ecole Nationale Supérieure des Mines de Paris, 1982.

[5] F. Meyer and J. Serra. Contrasts and activity lattice. Signal Processing, 16:303-317, 1989.

[6] C. Ronse and H. J. A. M. Heijmans. The algebraic basis of mathematical morphology - part II: Openings and closings. Computer Vision, Graphics and Image Processing: Image Understanding, 54:74-97, 1991.

[7] J. Serra, editor. Image Analysis and Mathematical Morphology. II: Theoretical Advances. Academic Press, London, 1988. 